\title{
FILTERING INSTAGRAM HASHTAGS THROUGH CROWDTAGGING AND THE HITS ALGORITHM
}

\author{
KANTHETI VENKATA MAHESH \\ PG Scholar, Department of Computer Science, \\ SVKP \& Dr K S Raju Arts \& Science College, \\ Penugonda, W.G.Dt., A.P, India. \\ venkatmaheshnanda341@gmail.com
}

\author{
CHIRAPARAPU SRINIVASA RAO* \\ Associate Professor in Computer Science, \\ SVKP \& Dr K S Raju Arts \& Science College, \\ Penugonda, W.G.Dt., A.P, India \\ chiraparapu@gmail.com
}

\begin{abstract}
Instagram is a rich source for mining descriptive tags for images and multimedia in general. The tags-image pairs can be used to train automatic image annotation (AIA) systems in accordance with the learning by example paradigm. In previous studies, we had concluded that, on average, $20 \%$ of the Instagram hashtags are related to the actual visual content of the image they accompany, i.e., they are descriptive hashtags, while there are many irrelevant hashtags, i.e., stop-hashtags, that are used across totally different images just for gathering clicks and for searchability enhancement. In this paper, we present a novel methodology, based on the principles of collective intelligence that helps in locating those hashtags. In particular, we show that the application of a modified version of the well-known hyperlinkinduced topic search (HITS) algorithm, in a crowdtagging context, provides an effective and consistent way for finding pairs of Instagram images and hashtags, which lead to representative and noise-free training sets for content-based image retrieval. As a proof of concept, we used the crowdsourcing platform Figure-eight to allow collective intelligence to be gathered in the form of tag selection (crowdtagging) for Instagram hashtags. The crowdtagging data of Figure-eight are used to form bipartite graphs in which the first type of nodes corresponds to the annotators and the second type to the hashtags they selected. The HITS algorithm is first used to rank the annotators in terms of their effectiveness in the crowdtagging task and then to identify the right hashtags per image.
\end{abstract}

Keywords: Bipartite graphs, collective intelligence, crowdtagging, FolkRank, hyperlinkinduced topic search (HITS) algorithm, image retrieval, image tagging, Instagram hashtags. 


\section{INTRODUCTION}

SOCIAL media are online communication channels dedicated to community-based input, interaction, content sharing, and collaboration. These media give the users the opportunity to share their content such as text, video, and images. Users usually accompany the content they post with text such as comments or hashtags. This alternative text (comment, hashtags, etc.) provides valuable information about the user posts and other information. Preece et al. construct a Sentinel platform that can enhance social media data in order to understand different situations they based also in Youtube video comments. Sagduyu et al. present a novel system that can present large-scale synthetic data from social media. In their system, they use textual content (hashtags and hyperlinks in tweets) to produce topics and trainthe n-gram model. The users in several of those media, e.g. Twitter, Instagram, and Facebook, use hashtags to annotate the digital content they upload. Hahshtags are, usually, words or nonspaced phrases preceded by the symbol \# that allow creators/content contributors to apply tagging that makes it easier for other users to locate their posts. A great portion of the digital content shared on social media platforms consists of images and short videos. Thus, effective retrieval of images from social media and the web, in general, becomes harder and more challenging day by day. Contemporary search engines are basically based on text descriptions to retrieve images; however, inaccurate text descriptions and the plethora of nontextually annotated images led to extended research for content-based image retrieval techniques.

The main problem of the content-based image retrieval is the so-called semantic gap: content-based retrieval is associated with low-level features while humans use high-level concepts for their search. To overcome this problem, automatic image annotation (AIA) methods were developed, that is, processes by which computing systems automatically assign metadata in the form of captions or keywords to images. Among the AIA methods, those based on the learning by example paradigm are probably the most common one. A small set of manually annotated training images are used to train models, which learn the correlation between image features and textual words (high-level concepts) and then allow automatic annotation of other (unseen) images. Obviously, good training examples, i.e., representative and accurate pairs of images and related tags are vital in this case. Social media, and especially the Instagram, provide a rich source of image-tag pairs. Mining the right ones, automatically or semi automatically, so as to be used as training examples is extremely important. We have to consider, however, that, in many cases, hashtags that accompany images in social media are not related with the image's content but serve several other purposes such as the expression of user's emotional state, the increase in user's clicks and findability, and the beginning of a new communication or discussion.

In our previous research, we have shown that the percentage of the Instagram hashtags that describe the visual content of the image they are associated with does not exceed $25 \%$ [12]. We have also noticed that many Instagram hashtags are used across images that have nothing in common, just for searchability enhancement. We named those hashtags as stop hashtags. Thus, filtering the Instagram hashtags in terms of the visual content of the image they accompany is 
required. Hyperlink-induced topic search (HITS) is a ranking algorithm than we could use to filter Instagram hashtags and locate the most relevant. The purpose of the HITS algorithm, developed by Jon Kleinberg, is to rate webpages. The basic idea is that a webpage can provide information about a topic and also relevant links for a topic. Thus, webpages belong to two groups: pages that provide good information about a topic ("authoritative") and those that give to the user good links about a topic ("hubs"). The HITS algorithm gives to each webpage both a hub and an authoritative value. We have started experimenting with the HITS algorithm for mining informative Instagram hashtags in one of our previous works and we extend this paper here by considering the application of the HITS algorithm in a real crowd tagging environment facilitated by the Figure-eight, formerly known as Crowd flower, crowdsourcing platform. In addition, we have increased the number of annotations per image to 500, we formed the bipartite graphs for all images, and we calculated the performance of annotators across all those images. Moreover, Folk Rank is used as a baseline to evaluate the performance of the proposed method.

\section{LITERATURE SERVEY}

\section{Topic modelling on Instagram hashtags: An alternative way to automatic image annotation}

Authors:Argyris Argyrou ; StamatiosGiannoulakis ; Nicolas Tsapatsoulis

\section{Abstract:}

Automatic Image Annotation (AIA) is the process of assigning tags to digital images without the intervention of humans. Most of the modern automatic image annotation methods are based on the learning by example paradigm. In those methods building the training examples, that is, pairs of images and related tags, is the first critical step. We have shown in our previous studies that hashtags accompanying images in social media and especially the Instagram provide a reach source for creating training sets for AIA. However, we concluded that only $20 \%$ of the Instagram hashtags describe the actual content of the image they accompany, thus, a series of filtering steps need to apply in order to identify the appropriate hashtags. In this paper we apply topic modelling with Latent Dirichlet Allocation (LDA) on Instagram hashtags in order to predict the subject of the related images. Since a topic is composed by a set of related terms, the identification of the visual topic of an Instagram image, through the proposed method, provides a plausible set of tags to be used in the context of training AIA methods.

\section{Crowdsourcing for multiple-choice question answering} Authors:Bahadir Ismail Aydin, Yavuz Selim Yilmaz, Yaliang Li, Qi Li, Jing Gao and Murat Demirbas

\footnotetext{
Abstract:

We leverage crowd wisdom for multiple-choice question answering, and employ lightweight machine learning techniques to improve the aggregation accuracy of crowdsourced answers to these questions. In order to develop more effective aggregation methods and evaluate them
} 
empirically, we developed and deployed a crowdsourced system for playing the "Who wants to be a millionaire?" quiz show. Analyzing our data (which consist of more than 200,000 answers), we find that by just going with the most selected answer in the aggregation, we can answer over $90 \%$ of the questions correctly, but the success rate of this technique plunges to $60 \%$ for the later/harder questions in the quiz show. To improve the success rates of these later/harder questions, we investigate novel weighted aggregation schemes for aggregating the answers obtained from the crowd. By using weights optimized for reliability of participants (derived from the participants' confidence), we show that we can pull up the accuracy rate for the harder questions by $15 \%$, and to overall $95 \%$ average accuracy. Our results provide a good case for the benefits of applying machine learning techniques for building more accurate crowd sourced question answering systems.

\section{Validity and reliability of naturalistic driving scene categorization judgments from crowdsourcing}

\section{Abstract}

A common challenge with processing naturalistic driving data is that humans may need to categorize great volumes of recorded visual information. By means of the online platform CrowdFlower, we investigated the potential of crowdsourcing to categorize driving scene features (i.e., presence of other road users, straight road segments, etc.) at greater scale than a single person or a small team of researchers would be capable of. In total, 200 workers from 46 different countries participated in 1.5 days. Validity and reliability were examined, both with and without embedding researcher generated control questions via the CrowdFlower mechanism known as Gold Test Questions (GTQs).

By employing GTQs, we found significantly more valid (accurate) and reliable (consistent) identification of driving scene items from external workers. Specifically, at a small scale CrowdFlower Job of 48 three-second video segments, an accuracy (i.e., relative to the ratings of a confederate researcher) of $91 \%$ on items was found with GTQs compared to $78 \%$ without. A difference in bias was found, where without GTQs, external workers returned more false positives than with GTQs. At a larger scale CrowdFlower Job making exclusive use of GTQs, 12,862 three-second video segments were released for annotation. Infeasible (and self-defeating) to check the accuracy of each at this scale, a random subset of 1012 categorizations was validated and returned similar levels of accuracy (95\%).

\section{EXISTING SYSTEM}

In this paper author is analyzing or filtering instagram hashtags given by crowds to detect whether hash tag is correct or not which is given by crowds. To identify correctness of tags author is using HIT algorithm. Now-a-days online social network users are posting messages with related pictures and the hash tags will be assigning to that picture. This related hash tags make other users to search that image easily. 
Sometime some user's assigns unrelated hash tags to images which make searching process difficult. To overcome from this issue author has introduce hash tags filtering technique using which we will filter hash tags to determine whether hash tag is relevant or irrelevant by matching content of both main hash tag and the annotator hash tags. If annotator assigns related hash tags then it will be relevant and supervisor will give good score to that annotator.

Using HIT algorithm we can determine whether that hash tags is used more frequently or not, if it's less frequent or unrelated hash tag then we will consider as stop hash tag.

In this application we used same given in paper to execute existing and extension logic. Existing technique will analyze given tags and annotators tags to find out whether given hashtag is relevant or irrelevant by using HIT algorithm. Our extension algorithm is based on Deep Learning Convolution Neural Network which analyze input image and describe contents available in image and then check whether extracted content from image and annotator's attribute are correct or not.

\section{PROPOSED SYSTEM}

In paper only one image used so we are also using same image but our extension technique will extract content from any image but for existing technique in paper author has expose only image details so we used same image

In our Application we are uploading one image and by seeing that image anybody can say that cat or kitten sitting on a bed with some stuff and our extension will describe same sentence or extract same data from image but existing technique just will check whether given hash tag and annotator tags are similar or relevant or not relevant.

\section{SYSTEM ARCHITECTURE}

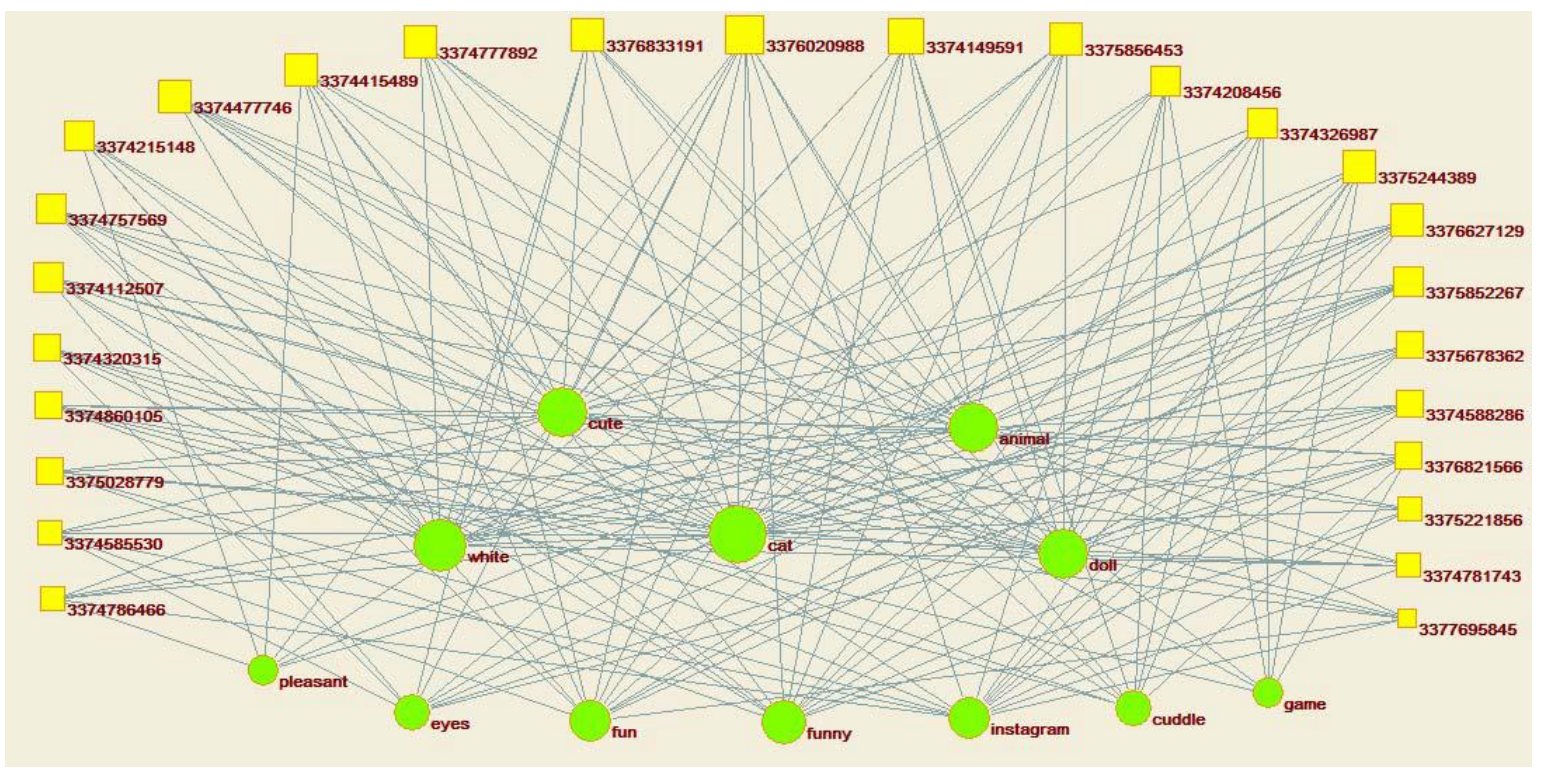

Subgraph of user-tag bipartite network for image \#7. Circles: tags. Boxes: annotators that selected those tags. 
In this paper author is analyzing or filtering instagram hashtags given by crowds to detect whether hash tag is correct or not which is given by crowds. To identify correctness of tags author is using HIT algorithm. Now-a-days online social network users are posting messages with related pictures and the hash tags will be assigning to that picture. This related hash tags make other users to search that image easily.

Sometime some user's assigns unrelated hash tags to images which make searching process difficult. To overcome from this issue author has introduce hash tags filtering technique using which we will filter hash tags to determine whether hash tag is relevant or irrelevant by matching content of both main hash tag and the annotator hash tags. If annotator assigns related hash tags then it will be relevant and supervisor will give good score to that annotator.

Using HIT algorithm we can determine whether that hash tags is used more frequently or not, if it's less frequent or unrelated hash tag then we will consider as stop hash tag.

In this application we used same given in paper to execute existing and extension logic. Existing technique will analyze given tags and annotators tags to find out whether given hashtag is relevant or irrelevant by using HIT algorithm. Our extension algorithm is based on Deep Learning Convolution Neural Network which analyze input image and describe contents available in image and then check whether extracted content from image and annotator's attribute are correct or not.

In paper only one image used so we are also using same image but our extension technique will extract content from any image but for existing technique in paper author has expose only image details so we used same image

\section{OUTPUT RESULTS}

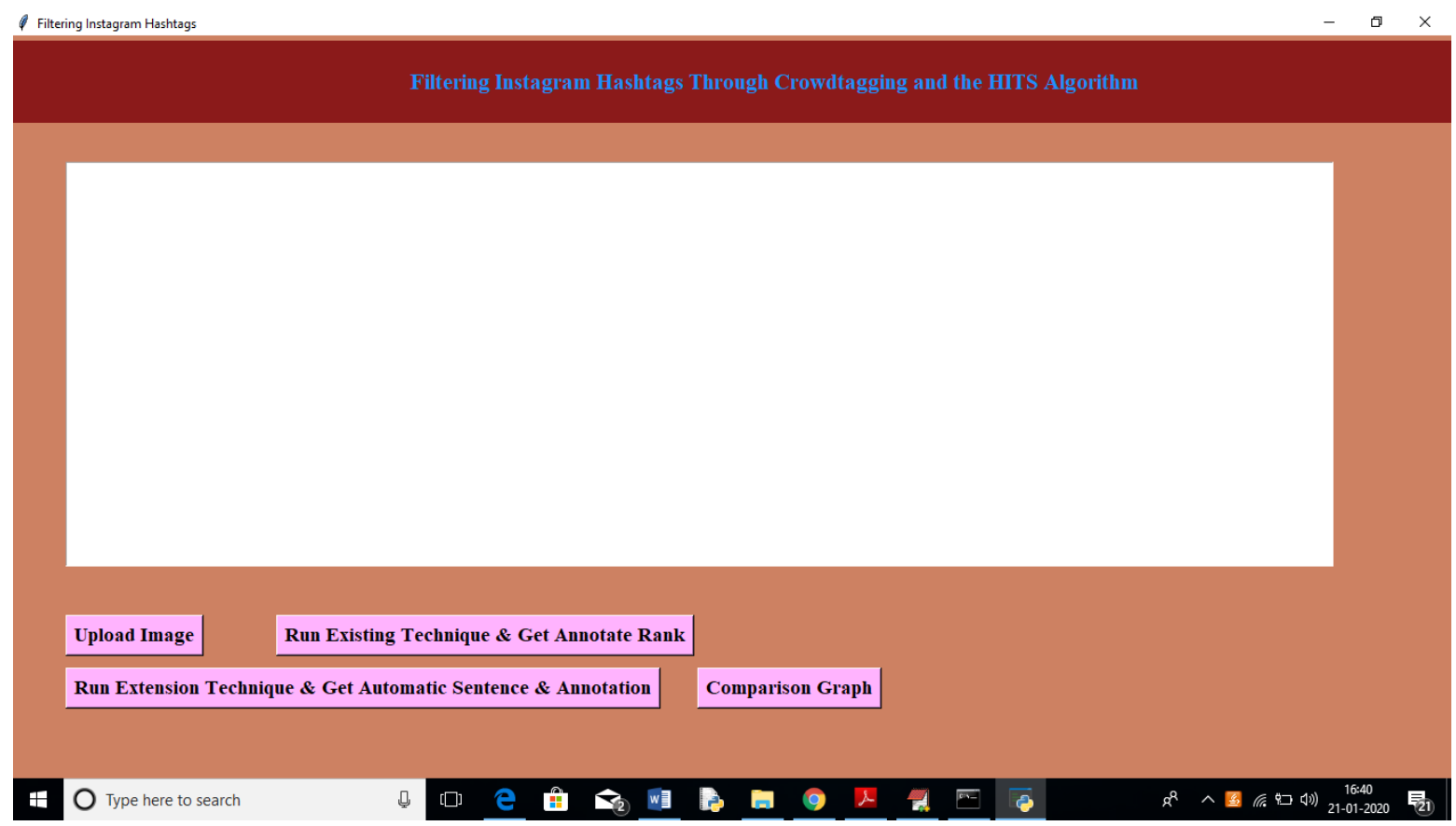

In above screen click on 'Upload Image' button to upload image 


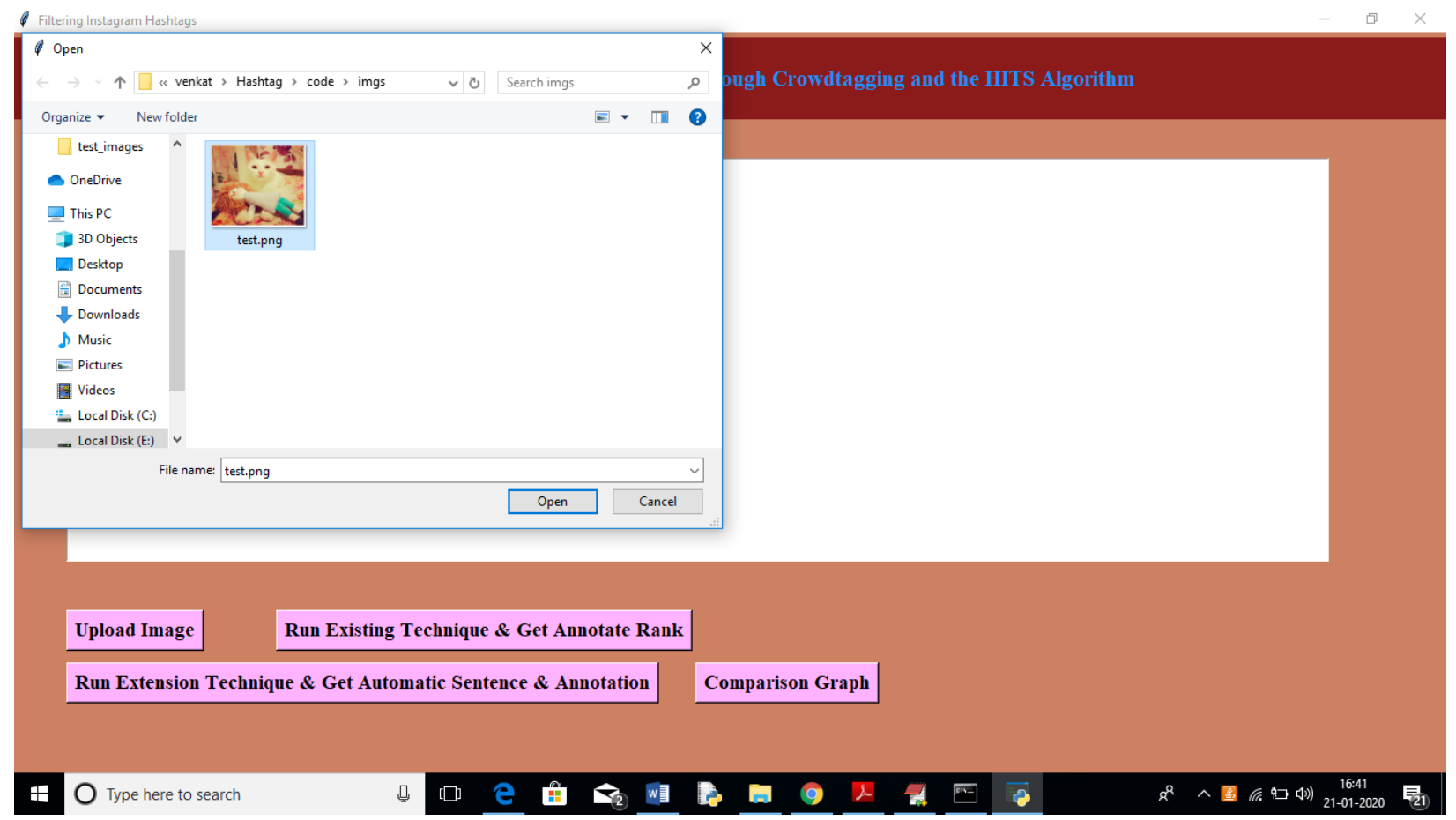

In above screen I am uploading one image and by seeing that image anybody can say that cat or kitten sitting on a bed with some stuff and our extension will describe same sentence or extract same data from image but existing technique just will check whether given hash tag and annotator tags are similar or relevant or not relevant. After uploading image will get below screen

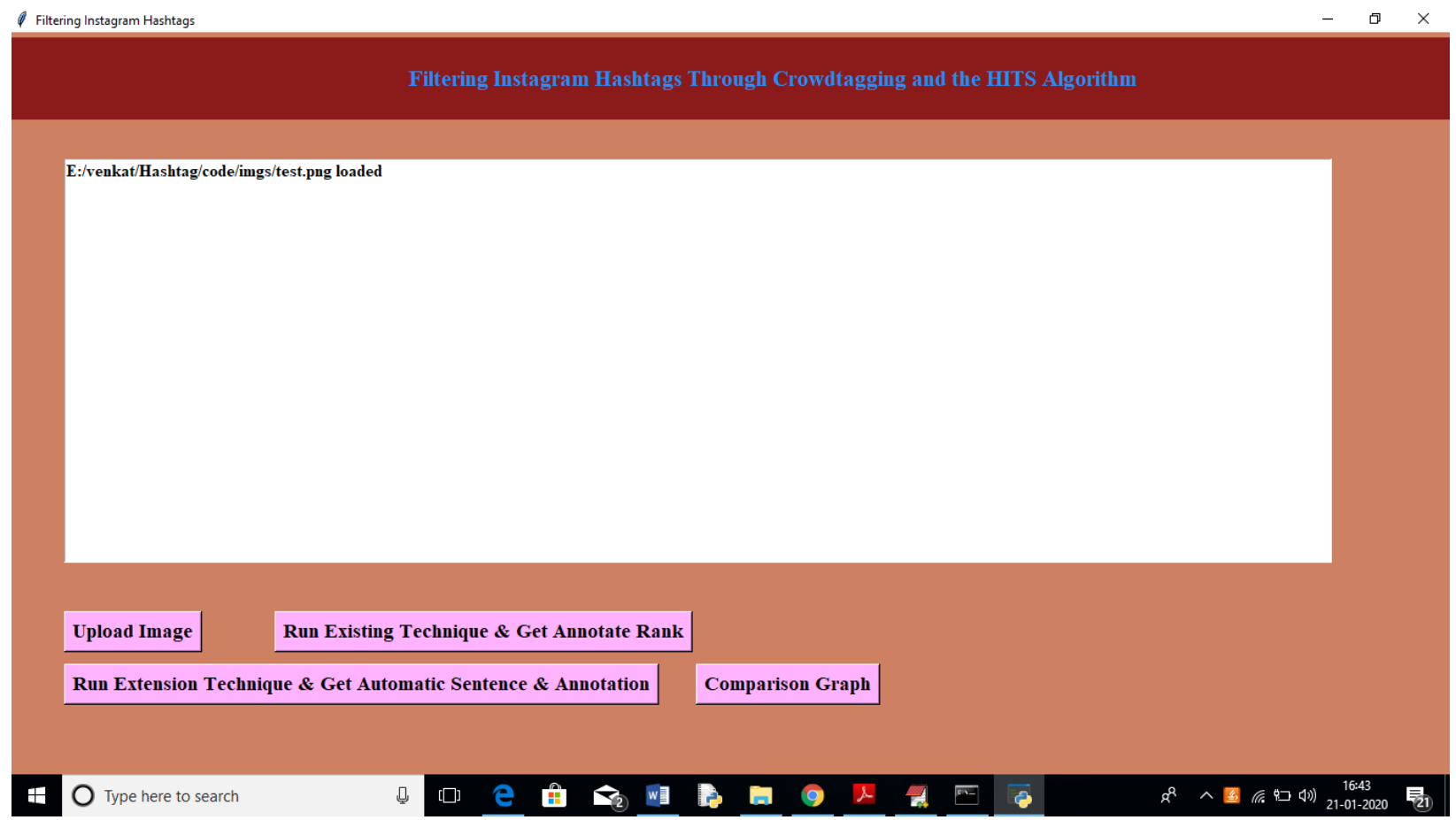

Now click on 'Run Existing Technique \& Get Annotation Rank' button to get below screen 


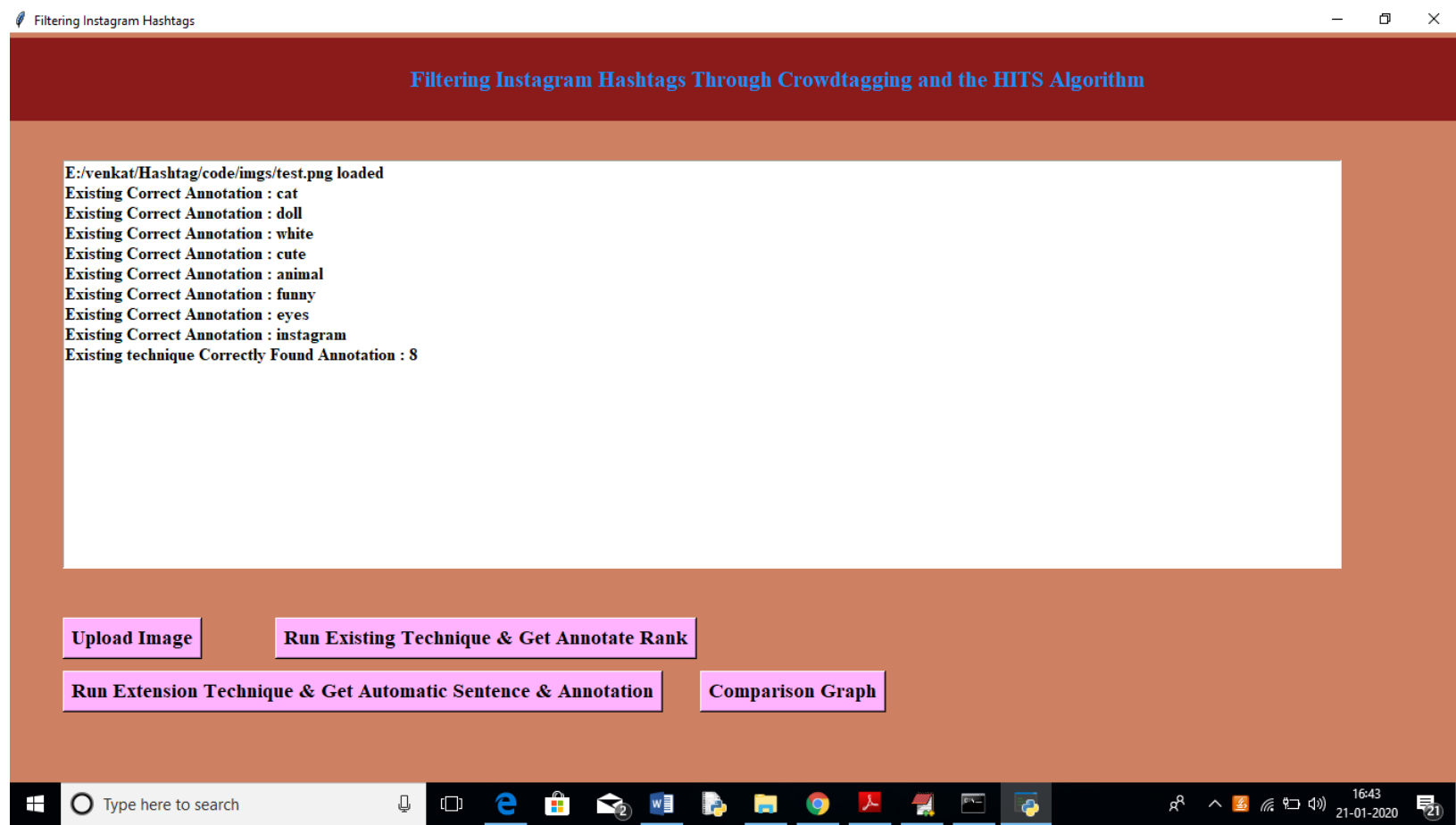

In above screen we can see from loaded images above annotations are correct as image contains cat, doll, cute etc. Existing technique able to extract 8 correct annotation from all annotated text. Now click on 'Run Extension Technique \& get Automatic Sentence \& Annotation' button to describe image in sentence and to check extracted words are matching with annotators words or not.

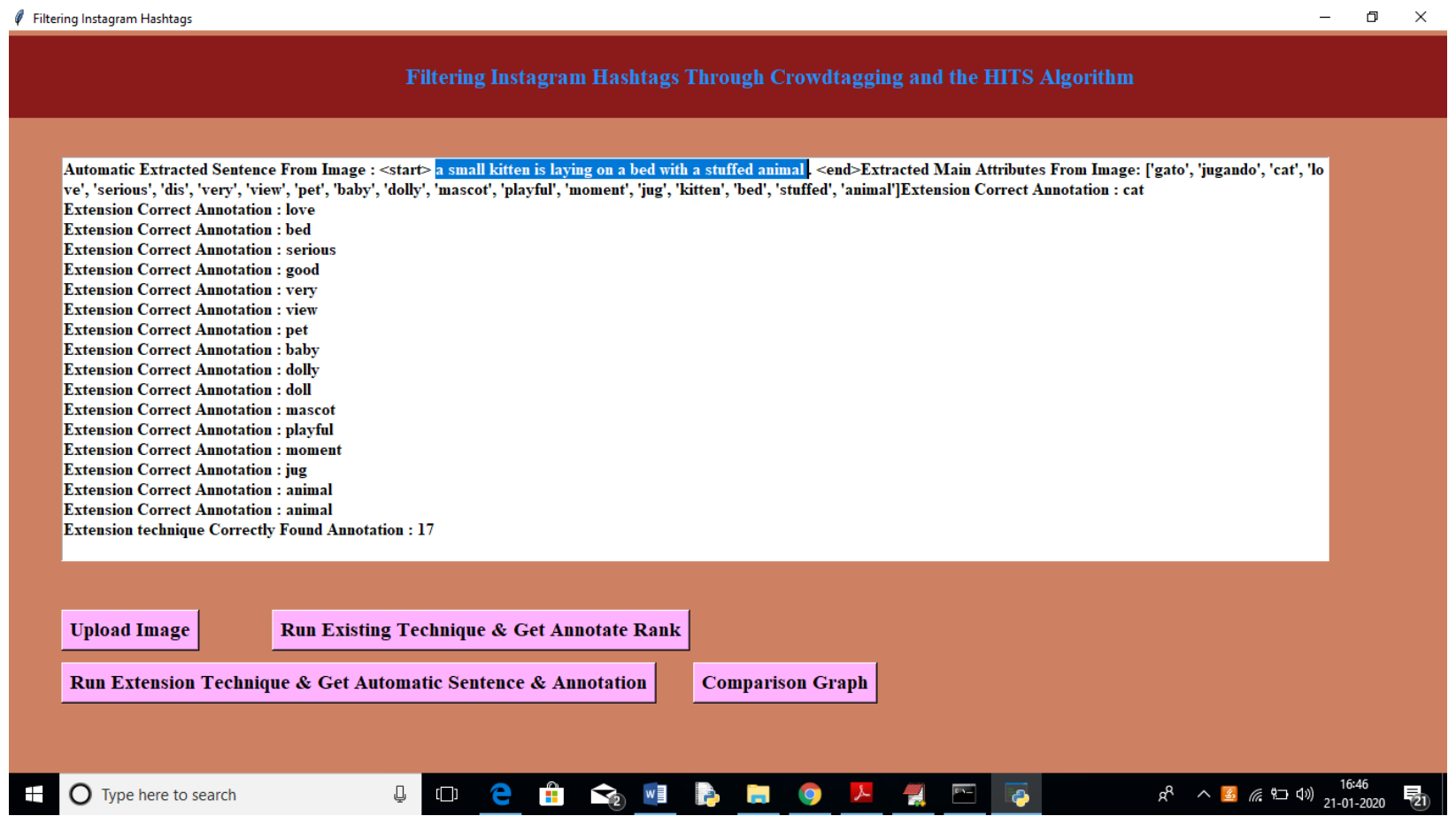

In above screen in selected text you can see our extension technique describing image in a sentence and then extracting words from image and compare with annotator's tags to get relevant 
details. Extension technique able to extract 17 related annotations. Now click on 'Comparison Graph' button to get below graph

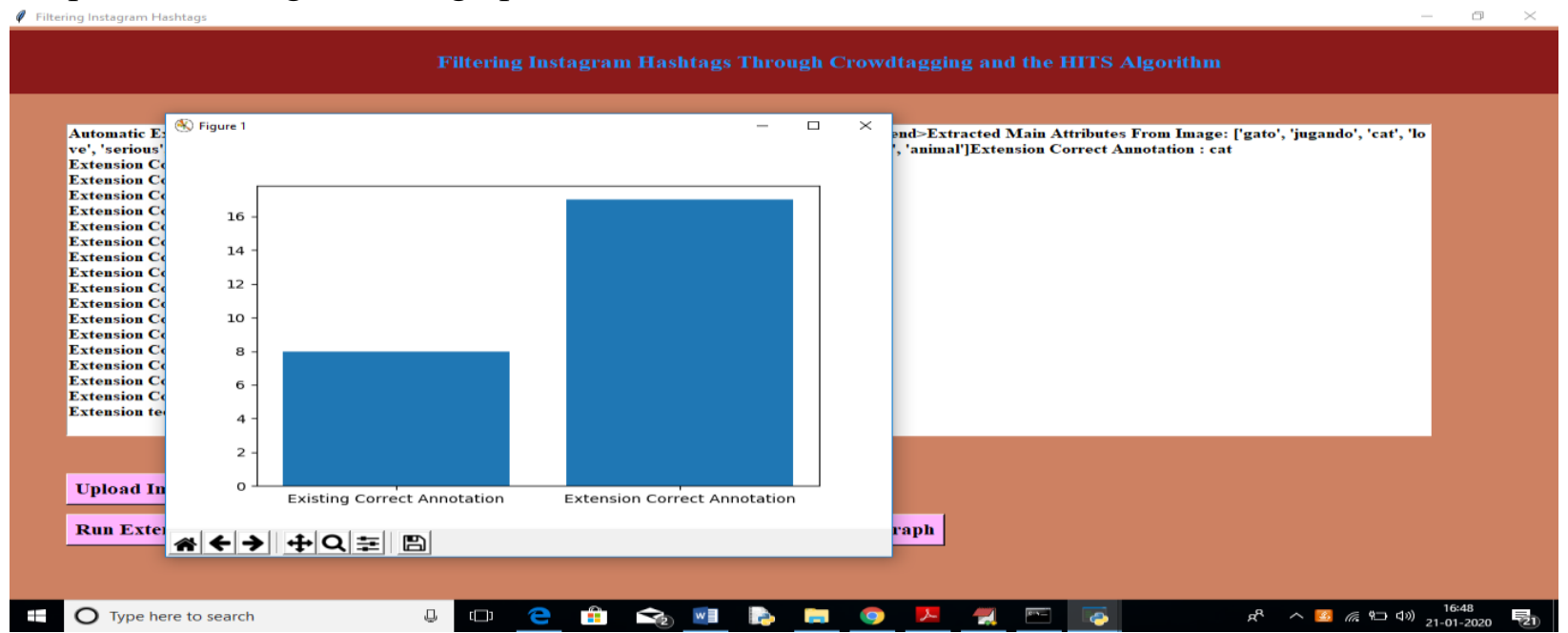

In above graph $\mathrm{x}$-axis represents technique name and $\mathrm{y}$-axis represents count of extracted matching annotations and we can see extension technique able to extract more related words compare to existing technique.

Note: existing technique can able to check with only one image as author given only one image details in paper and what other images he has used that information is not available. But extension technique can work with any image. See another image example

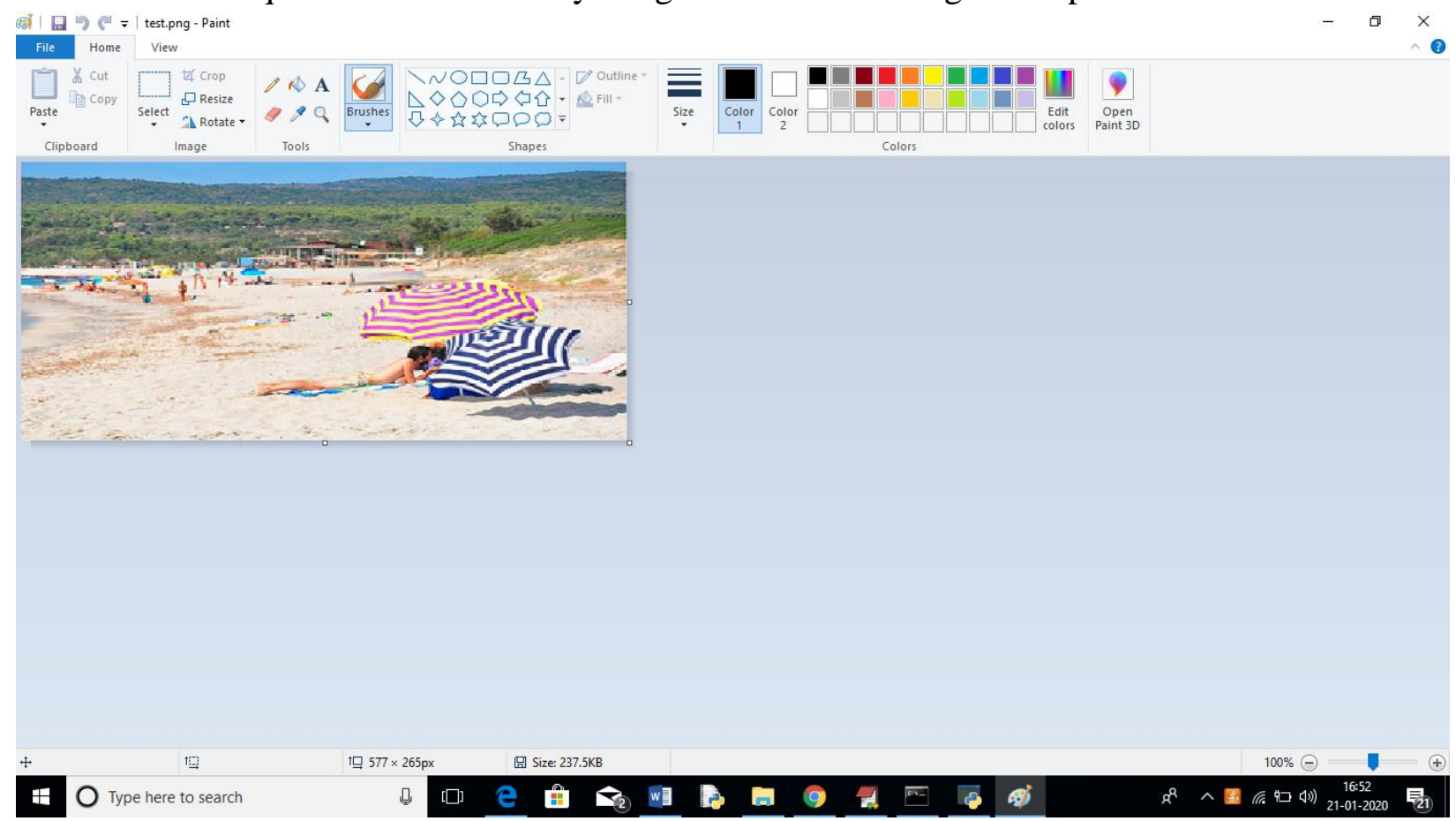

In above image we can see peoples are on beach with umbrellas and extension technique can extract this information but cannot compare with existing technique as author not include this image in his annotation dataset 


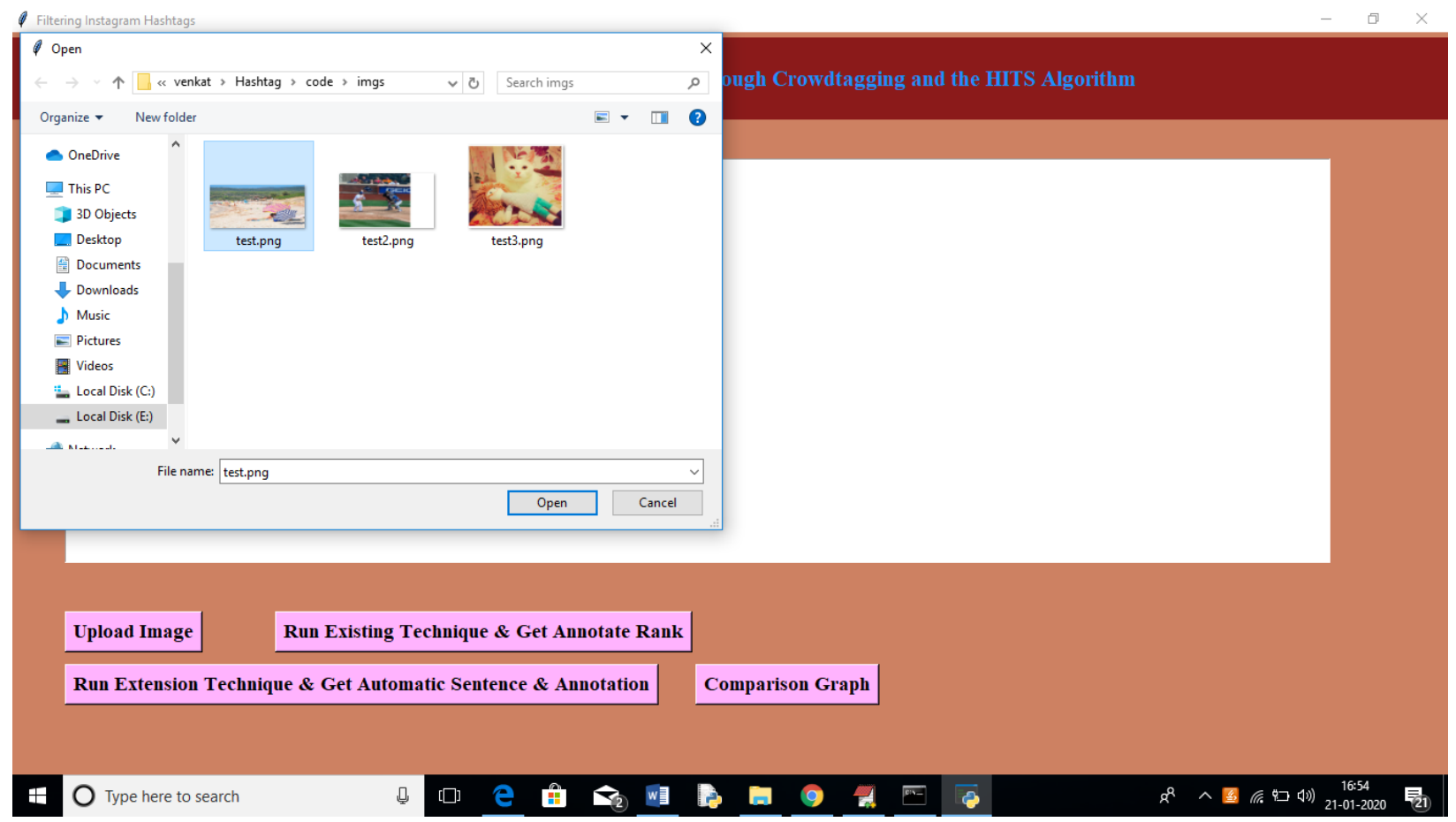

In above screen uploading same image and then click on 'Run Extension Technique \& get Automatic Sentence \& Annotation' button to get below results

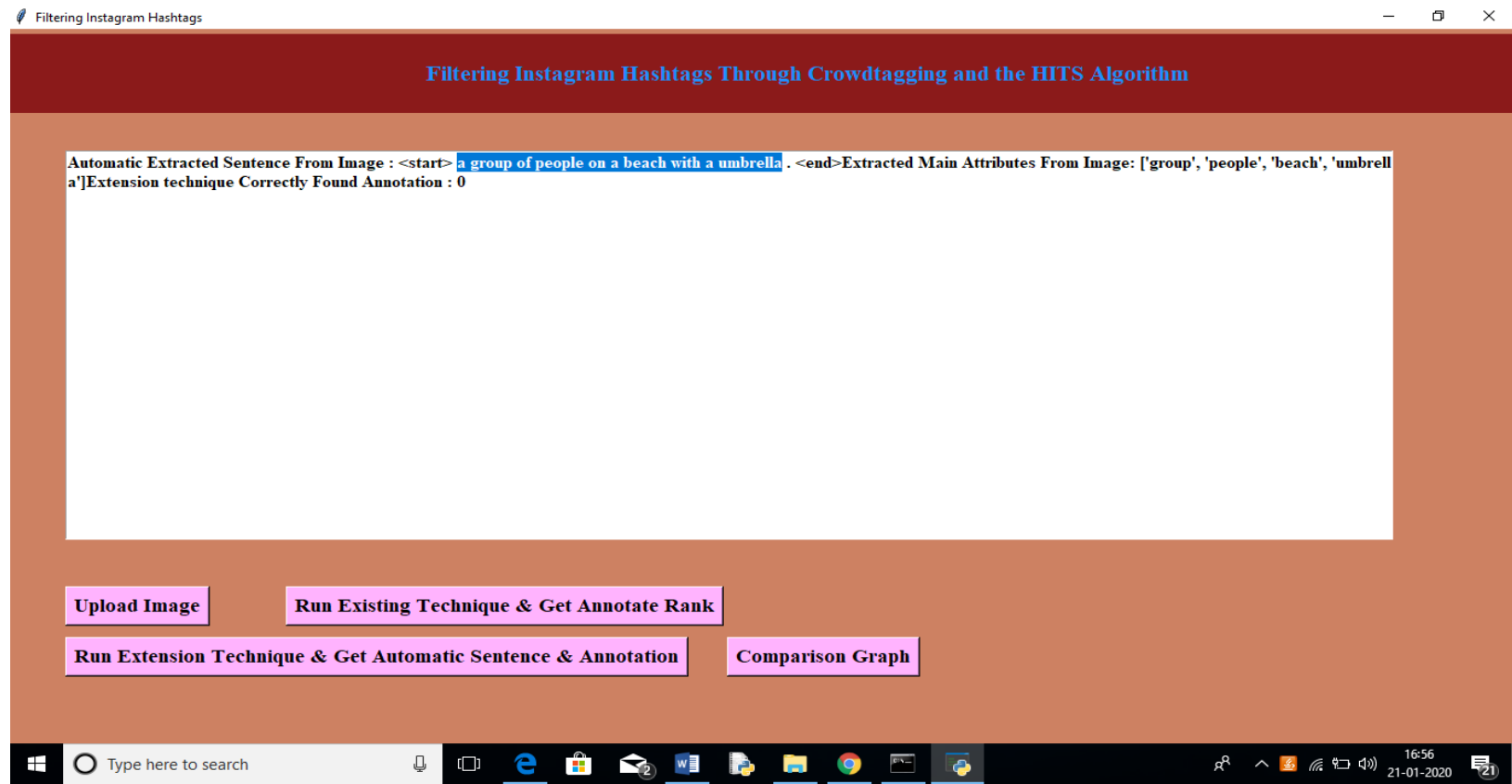

In above screen in selected text you can see sentence describing image and its related attributes or hashtag also displaying

\section{DISCUSSION AND CONCLUSION}

In this paper, we have presented an innovative methodology, based on the HITS algorithm and the principles of collective intelligence, for the identification of Instagram hashtags that describe 
the visual content of the images they are associated with. We have empirically shown that the application of a two-step HITS algorithm in a crowdtagging context provides an easy and effective way to locate pairs of Instagram images and hashtags that can be used as training sets for content-based image retrieval systems in the learning by example paradigm. As a proof of concept, we have used 25000 evaluations (500 annotations for each one of 50 images) collected from the Figure-eight crowdsourcing platform to create a bipartite graph composed of users (annotators) and the tags they selected to describe the 50 images. The hub scores of the HITS

Algorithm applied to this graph, called hereby full bipartite graph, give us a measure of the reliability of the annotators. The aforementioned approach is based on the findings of Theodosiou et al. [39], in which the reliability of annotators is better approximated if we consider all the annotations they have performed rather than the subset of gold test questions. In the second step, a weighted bipartite graph for each image is composed in the same way as the full bipartite graph. The weights of these graphs are the hub scores computed in the previous step. By thresholding the authority scores of the per image graphs, obtained by the application of the HITS algorithm on the weighted graphs, we can rank and then effectively locate the hashtags that are relevant to their visual content as per the annotators evaluation.

Some important findings of this paper are briefly summarized here. The first refers to the value of crowdtagging itself. In several studies before, we found that the crowd can substitute the experts in the evaluation of images with respect to relevant tags. However, even with a large number of annotators (499 in our case), it seems that a perfect agreement between annotators and experts cannot be achieved. In particular, it was found that from the 145 different tags suggested for the 50 images used in this paper by the two experts, only 135 were also identified by the 499 annotators. This leads to a maximum achievable recall value equal to 0.931 . Thus, in subjective evaluation tasks, such as those referring to the identification of tags that are related to the visual content of images, no perfect agreement between the experts and the crowd should be expected

A second finding is that crowd tagging of images can be effectively modeled through user-tag bipartite graphs, one per image. Thresholding the authority score of the HITS algorithm applied on these graphs is a robust way to identify the tags that characterize the visual content of the corresponding images. Getting the top ranked tags based on the authority score is an alternative solution, but, with a little bit lower effectiveness.

A final remark of this paper refers to the importance of using weighted user-tag bipartite graphs for the crowd tagged images. It appears that weighting the bipartite graphs with the hub scores of the annotators provides the best results. However, even in the case that the reliability metric of the crowdsourcing platform itself (the _trust variable of Figure-eight in our case) is used to weight the bipartite graphs, the results are not significantly worse. We are a little bit reluctant to generalize this conclusion because, in this paper, we have used too many annotations (499) per image. Thus, one of our future tests will involve a more typical image crowd tagging scenario in which much more images will be used and much fewer (typically less than five) annotations per image will be considered. In that case, only partial coannotation of the same images by the same annotators will take place in contrast to this paper in which all annotators annotated all images. 
We are currently working to check, in practice, that the image-hashtags pairs mined from the Instagram through the approach described in this paper can be used, indeed, for a large-scale AIA in a content-based image retrieval scenario as proposed by Theodosiou and Tsapatsoulis

\section{REFERENCES}

1.A. Argyrou, S. Giannoulakis and N. Tsapatsoulis, "Topic modelling on Instagram hashtags: An alternative way to automatic image annotation?", Proc. 13th Int. Workshop Semantic Social Media Adaptation Personalization, pp. 61-67, 2018.

2.B. I. Aydin, Y. S. Yilmaz, Y. Li, Q. Li, J. Gao and M. Demirbas, "Crowdsourcing for multiplechoice question answering", Proc. 28th. AAAI Conf. Artif. Intell., pp. 2946-2953, 2014.

3.C. D. D. Cabrall et al., "Validity and reliability of naturalistic driving scene categorization judgments from crowdsourcing", Accident Anal. Prevention, vol. 114, pp. 25-33, May 2018.

4.Q. Cheng, Q. Zhang, P. Fu, C. Tu and S. Li, "A survey and analysis on automatic image annotation", Pattern Recognit., vol. 79, pp. 242-259, Jul. 2018.

5.N. Craswell, "Mean reciprocal rank" in Encyclopedia of Database Systems, London, U.K.:Springer, pp. 1703, 2009.

6.H. Cui, Q. Li, H. Li and Z. Yan, "Healthcare fraud detection based on trustworthiness of doctors", Proc. Trustcom/BigDataSE/I SPA, pp. 74-81, 2016.

7.A. R. Daer, R. Hoffman and S. Goodman, "Rhetorical functions of hashtag forms across social media applications", Proc. 32nd ACM Int. Conf. Design Commun. CD-ROM, 2014.

8.E. Ferrara, R. Interdonato and A. Tagarelli, "Online popularity and topical interests through the lens of instagram", Proc. 25th ACM Conf. Hypertext Social Media, pp. 24-34, 2014.

9.J. M. Fletcher and T. Wennekers, "From structure to activity: Using centrality measures to predict neuronal activity", Int. J. Neural Syst., vol. 28, no. 2, 2018.

10.M. Gao, L. Chen, B. Li, Y. Li, W. Liu and Y.-C. Xu, "Projection-based link prediction in a bipartite network", Inf. Sci., vol. 376, pp. 158-171, Jan. 2017.

11.S. I. Gass and C. M. Harris, "Bipartite graph" in Encyclopedia of Operations Research and Management Science, Boston, MA, USA:Springer, pp. 126, 2013.

12.S. Giannoulakis and N. Tsapatsoulis, "Evaluating the descriptive power of instagram hashtags", J. Innov. Digit. Ecosyst., vol. 3, no. 2, pp. 114-129, 2016.

13.S. Giannoulakis and N. Tsapatsoulis, "Defining and identifying stophashtags in instagram", Proc. INNS Conf. Big Data, pp. 304-313, 2016.

14.S. Giannoulakis, N. Tsapatsoulis and K. Ntalianis, "Identifying image tags from Instagram hashtags using the HITS algorithm", Proc. 3rd Int. Conf. Big Data Intell. Comput. Cyber Sci. Technol. Congr. (DASC/PiCom/DataCom/CyberSciTech), pp. 89-94, 2017.

15.M. V. Giuffrida, F. Chen, H. Scharr and S. A. Tsaftaris, "Citizen crowds and experts: Observer variability in image-based plant phenotyping", Plant Methods, vol. 14, no. 1, pp. 12, 2018.

16.M. Gupta, R. Li, Z. Yin and J. Han, "Survey on social tagging techniques", SIGKDD Explor. Newslett., vol. 12, no. 1, pp. 58-72, Jun. 2010. 